Case Report

\title{
Abdominal Aortic Pseudo-Aneurysm, a Dreadful Complication of Caries Spine - A Case Report
}

\author{
Shams-ud-Din, Ishfaq Ahmed \\ Quaid-e-Azam International Hospital, Islamabad - Pakistan
}

\begin{abstract}
The thoracic spine is the most frequently involved region in carries spine, 60 percent; and in the thoracic spine, more than 70 percent occur in the midthoracic regions D8, 9, 10. The cauda equine and lumbosacral involvement frequency are less frequently involved. But the vascular complication it causes is a rare disease, with high mortality when it involves adjacent critical vascular structures like the abdominal aorta with complicating aneurysm and pseudoaneurysm. Evaluating a patient with a caries spine starts with a thorough history, physical examination findings, laboratory investigations like CBC, ESR, CRP, ICT TB, etc., complete radiological workup like $X$-rays, 3-D CT reconstruction of the whole spine, MRI whole spine with and without contrast. We presented a case of 21 years male who was suffering from caries spine, at D11 - D12; which had eroded his abdominal aorta secondary to bony spicules of carries. This erosion of the aorta had blood leak into psoas muscle, which was mimicking psoas abscess on preoperative CT scan spine, and MRI whole spine. During corrective surgery for his caries spine, unexpected torrential bleeding was encountered, when the left psoas muscle was given nick. Bleeding was stopped with packs and with the help of a cardiac surgeon. Postoperatively the patient was kept in ICU, but unfortunately on the $8^{\text {th }}$ post-surgery patient succumbs to his illness. In our case, psoas muscle pseudoaneurysm was missed radiologically in both MRI and CT spine preoperatively. Angiography with endovascular aortic stenting would have changed the surgical course of the patient.
\end{abstract}

Keywords: Dorsal spine D, Tuberculosis TB, complete blood count CBC, Erythrocyte sedimentation ESR, Computed tomography $\mathrm{CT}$, Magnetic resonance imaging $\mathrm{MRI}$, World health organization $\mathrm{WHO}$, Antituberculous treatment ATT.

Corresponding Author: Shams-ud-Din

Quaid-e-Azam International Hospital, Islamabad - Pakistan Email: asz-9-0@hotmail.com

Date of Submission: 03-09-2021

Date of Revision: 30-09-2021

Date of Acceptance: 30-09-2021

Date of Online Publishing: 30-09-2021

Date of Print: 30-09-2021

DOI: $10.36552 /$ pjns.v25i3.578

\section{INTRODUCTION}

Since ancient times, TB is one of the challenges to treat contiguous diseases. Ancient Greek used the word Phthisis for TB, in Ancient Hebrew Schachepheth while in Ancient Rome it was called Tabes. $^{1-3}$ Until the 1700 s, physicians noticed paleness patients secondary to TB and named them The White Plaque. ${ }^{2-3}$ It was first Schonlien who gave the name of Tuberculosis to this 
crippling disease in $1834 .^{1,2}$ Scrofula was the name given to TB when it involves neck and lymph nodes, which was considered a different disease from TB in the lungs. ${ }^{1-3}$ It was the discovery of mycobacterium tuberculosis in 1882 , a bacterium that cause tuberculosis by Dr. Robert Koch. ${ }^{3-4}$ These days TB is named either pulmonary or extrapulmonary, when it involves lungs or another system of the body, respectively. ${ }^{5-6}$

In all cases of spinal tuberculosis, the source of infection is hematogenous dissemination from primary visceral tuberculosis. Caries spine can cause various complications, from spinal column deformity to neurological manifestation. Tuberculosis is still a challenging disease to treat since prehistoric era. ${ }^{1-4}$ Robert Koch in the year 1882, when he discovered a bacteria called Mycobacterium tuberculosis. ${ }^{1,2}$ Of all the osseous tuberculous patients, 20 percent of the patients suffer from vertebral tuberculosis with equal age and sex distribution. ${ }^{4-6}$ Tuberculous abdominal aortic aneurysm/pseudo-aneurysm is a very rare complication of carries spine..$^{7-8}$ The causation of carries spine is secondary to the hematogenous spread or via Batson's plexus, of the bacterium; Mycobacterium tuberculosis, a type of granulomatous disease. ${ }^{1-5}$ The spine column is infected by Mycobacterium tuberculosis in many ways. $^{6-9}$

\section{1 - Paradiscal Variety}

It can be a lesion that is paradiscal, central vertebral body involvement, anterior to the vertebral body, and/or appendiceal involvement. $^{5-6}$

\section{2 - Central Type}

The disc space is destroyed by paradiscal type lesion and due to scarce blood supply in disc space, is rapidly destructive. ${ }^{6-7}$ The central type involves the vertebral body, which results in deformation of the boney spine with collapse and compression, and secondary neurological injury. ${ }^{7-9}$

\section{3 - Anterior Type}

The destruction of the vertebral body in the anterior type is mainly in the subperiosteal layer below the anterior longitudinal ligament, resulting in the periarteritis and necrosis of bone with secondary compression. ${ }^{8-11}$

\section{4 - Appendiceal Type}

The spinous process, lamina, pedicles, and articular process are involved in the appendiceal variety of carries spine..$^{5-6}$

Spine column spondylitis can progress to degenerative spine disease which leads to spinal deformity, with the neurological deficit being considered the most severe complication. ${ }^{5-7}$ The neurological complication is quoted to be $15-40$ percent due to a variety of pathophysiological ways, which lead to paralysis in most cases. It can be in acute disease, granulomatous chronic disease with sequestration of the bone sequel, unstable diseased spine, and dural fibrotic changes. $^{3-5}$

This is very rare, that boney spur penetrates the anterior longitudinal ligament and causing injury to the aorta, which had been associated with difficulty to manage and very high mortality. ${ }^{8-12}$ Especially to note, when minor rent into aorta mimic psoas abscess, then even modern diagnostics MRI, and CT may miss accurate diagnosis. ${ }^{11,12}$ Aorta starts from the heart and then, is divided into four parts, ascending aorta, the arch of the aorta, descending thoracic aorta, and the abdominal aorta below the diaphragm. At the level of the L4 vertebra, the aorta divides into right and left common iliac arteries. In 2018, it was by Yan Liang et al, who measure the pedicle to aortic angle in Pott's scoliotic spine at D10 - L1, and found the aortic to left pedicle distance was larger (6.36 +/$0.77 \mathrm{~cm})$ and the angles were smaller $(-8+-$ 2.89)..$^{3-4}$ They also concluded that angular kyphosis shifts aorta anteromedially. ${ }^{3-6}$

Management of caries spine starts as soon as 
it is diagnosed. Antituberculous treatment regime specified in the WHO guidelines includes combination drugs, rifampicin, isoniazid, ethambutol, and pyrazinamide for two months, then rifampicin and isoniazid combine drug for 12 or 18 months. ${ }^{1-4}$ Surgical intervention is required when there is damage to the vertebral body, and/or collapse, progressive neurodeficits by compression of the cord due to sequestrum and abscess, and unstable spine. ${ }^{5}$ We present this case of aortic aneurysm secondary to carries spine of lower dorsal D11 to L1, to highlight the importance of carefully studying aorta in carries spine before surgically embarking on these patients, and to avoid catastrophic bleeding complications preoperatively. ${ }^{11,12}$

\section{CASE REPORT}

A 21 years male patient presented to the emergency department of Quaid-e-Azam International hospital Islamabad, on $14^{\text {th }}$ April 2021, with severe back pain, especially at the dorsal spine. The pain was gradual in onset, progressive for the last 3months. Severe in intensity with visual analog scale 8 , continuously worsening for last $7-8$ days. The pain was radiating to the chest and abdomen with progressive paraparesis for 4 days, and inability to walk with urinary and stool incontinence.

This patient was already diagnosed at some other institute for caries spine some 4 months back, along with the biopsy-proven pathology of tuberculosis of the spine. The patient was already taking ATT for the last 4months with no symptomatic improvement in pain and neurodeficits.

On examination, tender spine along D11, D12 - L3 noted. Neurological sensory deficit noted at D11 - D12 level and MRS scale 2/5 below D12. Patient blood tests and CT whole spine and MRI whole spine done in an emergency. CT and MRI spine show extensive carries at lower dorsal and upper lumbar spine along with partly calcified prevertebral and bilateral paravertebral abscess in bilateral psoas muscle extending down to LV5 on left. There was the partial destruction of D11, D12, LV1 bodies and complete necrosis of D12/LV1 intervertebral

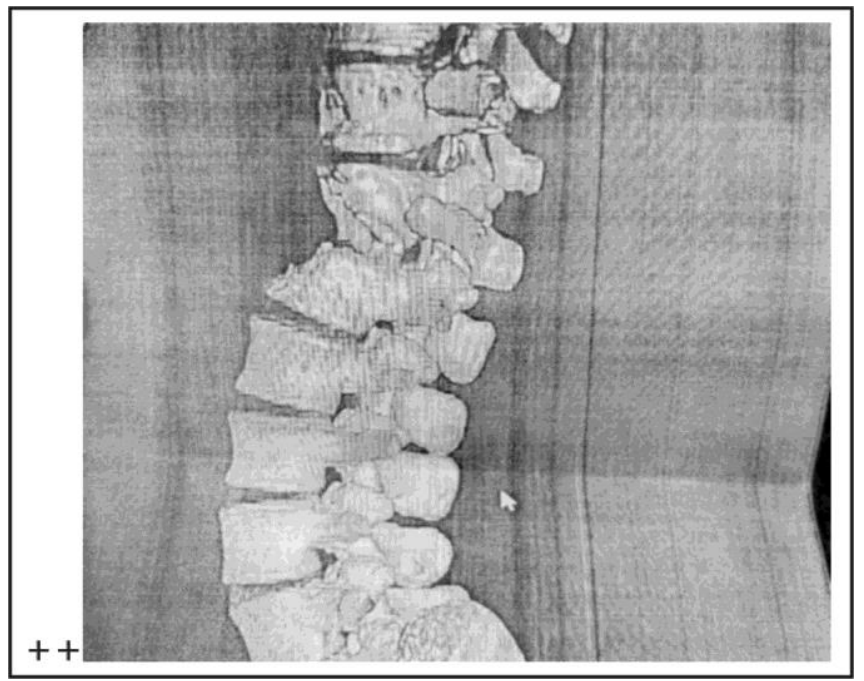

Figure 1: $C T$ 3-D Reconstruction of Dorsolumbar spine showing D12 - L1 erosions with kyphotic deformity. (The scan is included with the consent of the patient).

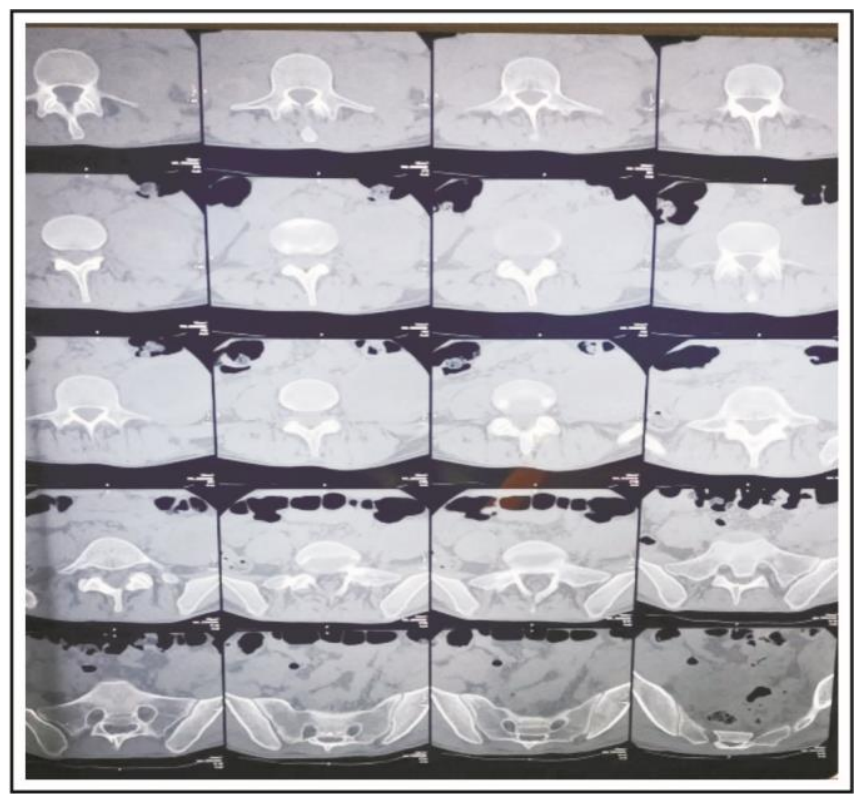

Figure 2: Axial cut CT dorsolumbar spine showing the complete collapse of D12-L1 with the compromised canal. (The scan is included with the consent of the patient). 


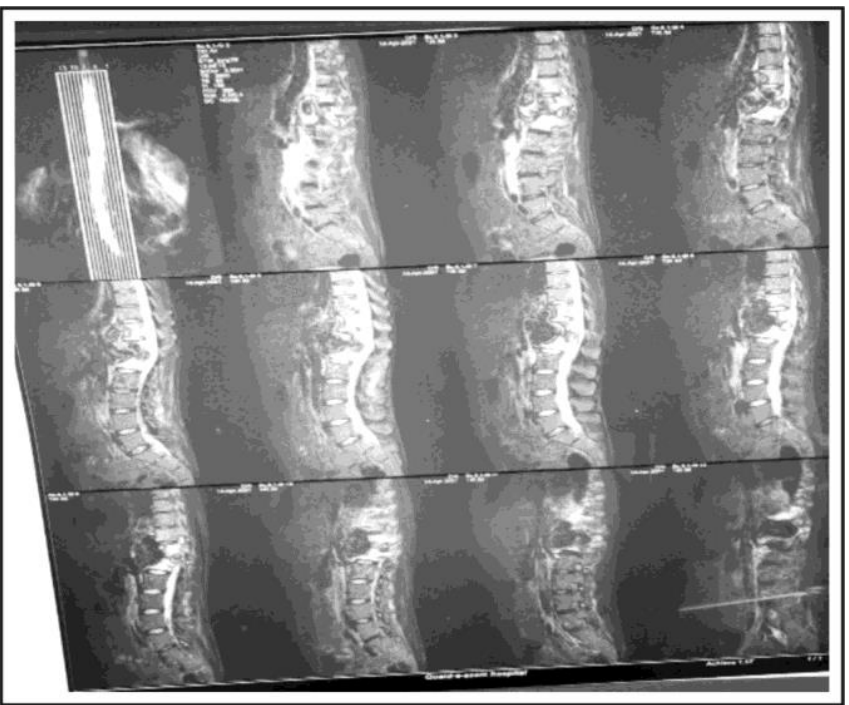

Figure 3: MRI T2 WT sagittal view bilateral psoas abscess from D11 extending up to $L 4-5$ with the destruction of D12 - L1 intervertebral disc, and thecal indentation with kyphotic deformity. (The scan is included with the consent of the patient).

disc with thecal sac indentation hyperintense T2 WT cord changes at D11 D12 along with kyphotic

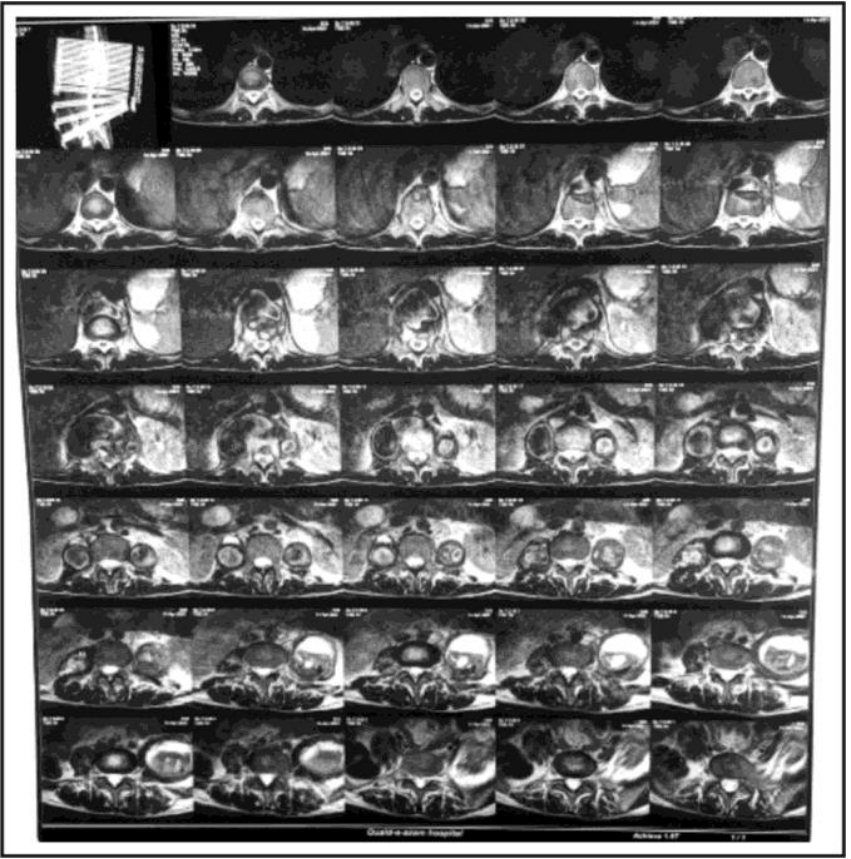

Figure 4: Axial $T_{2}$ WT MRI Dorsolumbar spine again showing bilateral psoas abscess from D11 through L4 5 with D12 - L1 collapse with thecal indentation and kyphotic deformity. (The scan is included with the consent of the patient). deformity at the same level. The patient was diagnosed to be suffering from extensive carries spine at D12/ L1. The patient was initially not fit for surgical intervention for the medical reason of anemia and a decrease BMI of 15 . It was decided to treat conservatively with ATT, dorsolumbar corset, and immobilization along with analgesia. His pain improved and discharged after 5 days in the hospital.

On the $3^{\text {rd }}$ post-discharge day, the patient presented with impending cauda equine. Patient and relatives were explained about the possible surgical intervention as no other option was available other than surgical intervention. Our goal of surgery was to drain the whole abscess in the psoas muscle, remove bone debris at D12/L1, irrigation, and remove infected degenerated intervertebral disc to relieve pressure from the thecal sac and spinal cord. Placement of Titanium cage implant along with rod and screw fixation from D11 to LV2, to achieve stability and alignment of the spinal column.

\section{Surgical Procedure}

The anesthesia team assesses the patient for GA fitness and it was a high-risk surgery. After highrisk consent from the patient and relatives, the patient was shifted to the operation theater. GA was given and the patient was positioned in the left up lateral position. Surgical marked site D11 L3 was cleaned and draped. D12/L1 site was approached by standard minimally invasive extracavitary retropleural/retroperitoneal technique (LECA). The left $11^{\text {th }}$ rib was resected as part of a surgical procedure for better exposure and its use as a bone graft inside the cage. The left psoas muscle was given nick for abscess drainage, but to our surprise, unexpected highpressure torrential hemorrhage was encountered, that was immediately controlled with packs and pressure. Our per operative diagnosis was that the abdominal aorta was eroded by carries bony spurs of D12/L1. Leakage of blood from this rent 
had turned both psoas muscle into the highpressure pseudoaneurysm. Meanwhile, cardiac surgeon and vascular surgeon were involved for further management of aortic rent, which was source of bleeding. Incision at the level of D7 given, thoracotomy done in order to achieve homeostasis and control source of bleeding. Left psoas incision stitched and bleeding secured, but during all this process of surgery, the patient went into hypovolemic shock, which was treated by anesthesia according to standard protocol.

Post surgically the patient was shifted to ICU for further management and planned for angiography once a patient is stable. During the ICU stay, the patient had developed coagulopathy due to massive blood transfusion, with deranged INR. Fresh frozen plasma and mega bags of platelets were transfused to correct coagulopathy. The patient remained in ICU for 2days and was treated according to standard protocol.

Patient relatives shifted the patient to a public hospital on $3^{\text {rd }}$ post-surgery day. Unfortunately, the patient succumbs to his illness on the $8^{\text {th }}$ postoperative day.

Tuberculous aortic pseudo-aneurysm associated with vertebral tuberculosis is a very rare complication of carries spine. In our case, psoas muscle pseudoaneurysm was missed radiologically in both $\mathrm{MRI}$ and $\mathrm{CT}$ spine preoperatively. Had it been not missed, angiography with endovascular aortic stenting would have changed the surgical course of the patient.

\section{DISCUSSION}

Globally it is estimated that tuberculosis is affecting 30 million people, with $1-3$ percent suffering skeletal TB, of which more than 50 percent are spinal tuberculosis. Spinal tuberculosis occurs with equal incidence in both age and sex. ${ }^{2-4}$ In almost all caries spine cases it is the hematogenous spread of the infection from other viscera, and in some cases, the spread can also be the Batson's plexus. Spinal column involvement can be paradiscal, central type, anterior, and/or, appendiceal. ${ }^{5-6}$ The aorta location anteriorly about $7 \mathrm{~cm}+-0.6 \mathrm{~cm}$ from left pedicle at D10 - L1, may mimics an aneurysm when the body of vertebrae collapses secondary to abscess of paravertebral tissues..$^{7-9}$ Shifting of thoracic aorta posteriorly in scoliotic spine very rarely may be involved by the bony spurs and abscess of carries. Only very few cases have been reported on this subject of transposition of thoracic aorta posteriorly than normal. ${ }^{9-11}$ At Cobb's angle < 70, aortic deflection occurs, and already diseases and deformed spine can involve the aorta, challenging management of such rare patients. ${ }^{1-12}$ The neurological complications of carries spine are well elaborated in the literature, but the vascular complication is scarcely mentioned. ${ }^{3-5}$ This kind of aortic aneurysms comes under the term of mycotic aneurysms ${ }^{9}$, with dreadful complications, especially when there is minor rent in the aneurysm, mimicking psoas abscess, as in our case. Uncommonly seen, the aortic wall may be involved and can result in the secular type of mycotic aneurysm. ${ }^{7-8}$ The aorta is involved by either hematogenous Vasavasorum involvement or contagious spread..$^{7-9}$ This becomes a real challenging situation to manage. ${ }^{11,12}$ More than 75 percent of caries spine patients had neurologic symptoms at presentation with bowel and bladder incontinence, paraplegia, and incapacitating pain. $^{6-9}$

Regarding radiological findings, X-rays will show reduce disc space, scalloping of vertebral bodies, and collapse of vertebral body. ${ }^{6-10}$ Vertebral body destruction leads to a deformed kyphotic spine with a decrease cobb's angle $<70$. CT 3-D reconstruction is excellent to assess the destruction of vertebrae, a paravertebral extension of lesion, and canal compromise. ${ }^{5-7} \mathrm{MRI}$ T2 or STIR (short T1 inversion recovery) is of great use for intradural and extradural invasion of carries spine, and contrast T1 MRI gives a clear 
picture of ring enhancement. ${ }^{7-11}$ If there is any suspicion of vascular involvement, it is advised to do angiography of great vessels and to do stenting preoperatively if the aneurysm is found..$^{9-12}$

The management starts with an Antituberculous treatment regime, specified in the WHO guidelines, including combination drugs, rifampicin, isoniazid, ethambutol, and pyrazinamide for two months then rifampicin and isoniazid combine drug for 12 or 18 months. ${ }^{1-5}$ Surgical intervention is required in cases for draining an abscess in psoas muscle, corpectomies, bony necrosis fragments and degenerated disc removal, relieving pressure from the thecal sac in the face of acute neurological deterioration, and placement of titanium cage implant along with rods and screws fixation for unstable deformed spine. .-8 $^{6-8}$

\section{CONCLUSION}

Great vessel involvement by caries dorsal spine is rarely described in the literature. A mycotic aortic aneurysm can occur in carries and angiography is a valuable investigation to be done preoperatively to avoid catastrophic preoperative bleeding complications, which then becomes extremely difficult to manage with very high mortality.

\section{REFERENCES}

1. Global Tuberculosis Report 2016. World health organization. Available at:

http://www.who.int/tb/publications/globalreport/en/ (Accessed on 06/12/2016).

2. Hayman J. Mycobacterium ulcerans: an infection from Jurassic time? Lancet. 1984; 2 (8410): 101516.
3. Zimmerman MR, Bull NY. Pulmonary and osseous tuberculosis in an Egyptian mummy. Acad Med. 1979; 55 (6): 604-08.

4. Turgut M. Spinal tuberculosis (pott's disease): its clinical presentation, surgical management, and outcome. A survey study on 694 patients. Neusurg Rev. 2001; 24: 8-13. (Pub Med)

5. Li FP, Wang XF, Xiao YB. Endovascular stent graft placement in the treatment of a ruptured Tuberculous pseudoaneurysm of descending thoracic aorta secondary to Pott's disease of spine. J Card Sug. 2012; 27: 75-7.

6. Yan Liang, Yongfei Zhao, Haing Liu, Zheng Wang. The position of the Aorta relative to the spine in patients with potts Thoracolumber angular kyphosis. Journal of Orthopaedic Science, March 2018; Vol. 23, Issue 2: pages: 289-93.

7. Kotil K, Alan MS, Bilge T. Medical management of Pott's disease in the thoracic and lumber spine: $A$ prospective clinical study. J Neurosurg Spine, 2007; 6: 222-8.

8. Golzarian J, Cheng J, Giron F, Bilfinger TV. Tuberculous pseudoaneurysm of the decending thoracic aorta: successful treatment by surgical excision and primary repair. Tex Heart Ins J. 1999; 26: 232-5.

9. Dahl T, Lange C, Odegard A, Berg K, Osen S, Myhre HO. Ruptured abdominal aortic aneurysm secondary to Tuberculous spondylitis. Int Angiol. 2005; 24: 98-101.

10. Hagino RT, Clagett GP, Valentine RJ. Acase of pott's disease of spine eroding into suprarenal aorta. J Vasc Surg. 1996; 24: 482-6.

11. Garg RK, Somvanshi DS. Spinal tuberculosis: A review. J Spinal Cord Med. 2011; 34: 440-54.

12. Falkensammer J, Behensky $H$, Gruber $H$, Prodinger WM, Fraedrich G. Successful treatment of a Tuberculous vertebral osteomyelitis eroding the Thoracoabdominal aorta: A case report. J Vas Surg. 2005; 42: 101-3. 


\section{Additional Information}

Disclosures: Authors report no conflict of interest.

Ethical Review Board Approval: The study was conformed to the ethical review board requirements.

Human Subjects: Consent was obtained by patient in this study.

Conflicts of Interest:

In compliance with the ICMJE uniform disclosure form, all authors declare the following:

Financial Relationships: All authors have declared that they have no financial relationships at present or within the previous three years with any organizations that might have an interest in the submitted work.

Other Relationships: All authors have declared that there are no other relationships or activities that could appear to have influenced the submitted work.

\section{AUTHORS CONTRIBUTIONS}

\begin{tabular}{|l|l|l|}
\hline Sr.\# & Author's Full Name & Intellectual Contribution to Paper in Terms of: \\
\hline 1. & Ishfaq Ahmed & Study Design and Methodology. \\
\hline 2. & Shams-ud-Din & Paper Writing, Referencing, Data Calculations and \\
\hline 3. & Shams-ud-Din & Data Collection and Calculations. \\
\hline 4. & Shams-ud-Din & Analysis of Data and Interpretation of Results etc. \\
\hline 5. & Ishfaq Ahmed & Literature Review and Manuscript Writing. \\
\hline
\end{tabular}

